\title{
Abscess resulting from Mycobacterium kansasii in the left thigh of AIDS patient ${ }^{*}$
}

\author{
Catarina Tenório de Lima ${ }^{1}$
}

Vera Magalhães ${ }^{1}$

\begin{abstract}
A case of abscess resulting from Mycobacterium kansasii, in the left thigh of a 53-year-old woman infected with the Human Immunodeficiency virus, is reported. Curiously, there was no pulmonary or systemic involvement as is usual with these Mycobacterium infections. The patient had CD4 T lymphocyte count of 257 cells $/ \mu \mathrm{L}$ and a viral load of 60,154 copies. Despite presenting a relatively preserved immunity, the patient also presented Criptococcic meningoencephalitis and Esophageal candidiasis. The patient responded satisfactorily to treatment for infections and after 51 days was discharged.
\end{abstract}

Keywords: Abscess; Acquired immunodeficiency syndrome; HIV; Mycobacterium kansasii

\section{INTRODUCTION}

Mycobacterium kansasii is an acid-fast bacillus, of slow growth, photochromogenic, yellow pigment producer when exposed to light. ${ }^{1}$ Described in 1953 by Buhler and Pollack, it is considered the most virulent of the non-tuberculous mycobacteria. ${ }^{2}$ They are widely distributed in water, biofilms, in the soil and in a great variety of animals. The asymptomatic infections are very common in human beings and may be acquired through cutaneous, respiratory, gastrointestinal and more rarely parenteral exposure.

Infection by $M$. kansasii in patients affected by the Human Immunodeficiency virus (HIV) is associated with advanced immunodeficiency, with CD4 T lymphocyte count below 100 cells/uL and is usually manifested by pulmonary lesions or disseminated disease. $^{3}$

It is the second most frequent non-tuberculous mycobacteria in patients with AIDS, being surpassed only by Mycobacterium avium-intracellulare Complex. ${ }^{4}$ We describe the first case in national literature of $M$. kansasii infection with extrapulmonary involvement exclusively.

\section{CASE REPORT}

A 53-year-old female patient was hospitalized in the infirmary at the teaching hospital of the Universidade Federal de Pernambuco, presenting a tumor in the left thigh $(15 \times 7 \mathrm{~cm})$, painful to the touch and increased temperature (Figure 1). She reported the onset of symptoms 4 months before, associated with weight loss $(8 \mathrm{~kg})$ and odynophagia. She denied fever. At the physical exam, she was conscious, oriented, with no visceromegalies. She presented white plaques in the oropharyngeal mucosa. Upper digestive endoscopy revealed esophagitis by Candida spp. A rapid HIV test was performed, which resulted positive. Treatment for esophageal candidiasis was then started with fluconazole $200 \mathrm{mg}$ / day. The initial CD4 T lymphocyte count was 257 cells $/ \mu \mathrm{L}$ and the viral load was 60,154 $(\log 4.779)$.

An ultrasound of the left thigh lesion was performed which revealed a voluminous heterogeneous collection of thickened and irregular walls with anechoic content, measuring $14.9 \times 7.0 \times 5.1 \mathrm{~cm}$, located in the subcutaneous cellular plane of anterior face of distal 1/3 of thigh (Figure 2). The magnetic resonance exam of the same lesion showed expansive formations

\footnotetext{
Received on 03.05.2013.

Approved by the Advisory Board and accepted for publication on 18.08.2013.

* Work performed at Hospital das Clínicas - Universidade Federal de Pernambuco (UFPE) - Recife (PE), Brazil.

Financial Support: none

Conflict of Interests: none

Universidade Federal de Pernambuco (Federal University of Pernambuco - UFPE) - Recife (PE), Brazil.

C 2014 by Anais Brasileiros de Dermatologia
} 


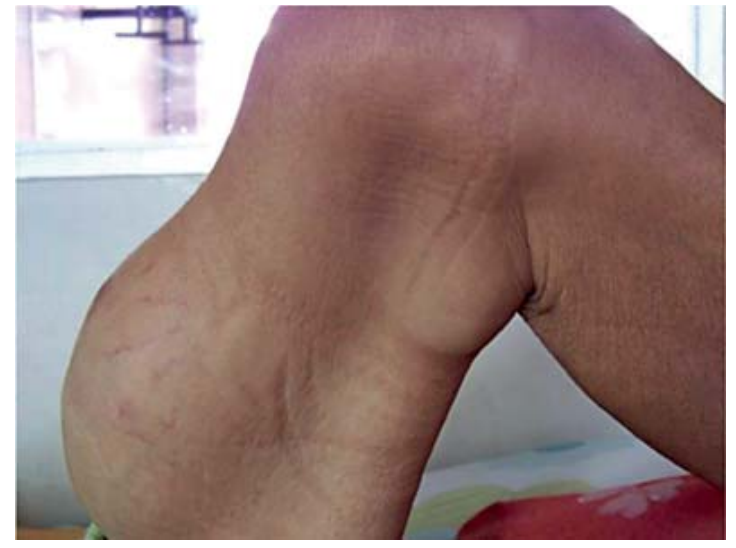

Figure 1: Abscess in thigh

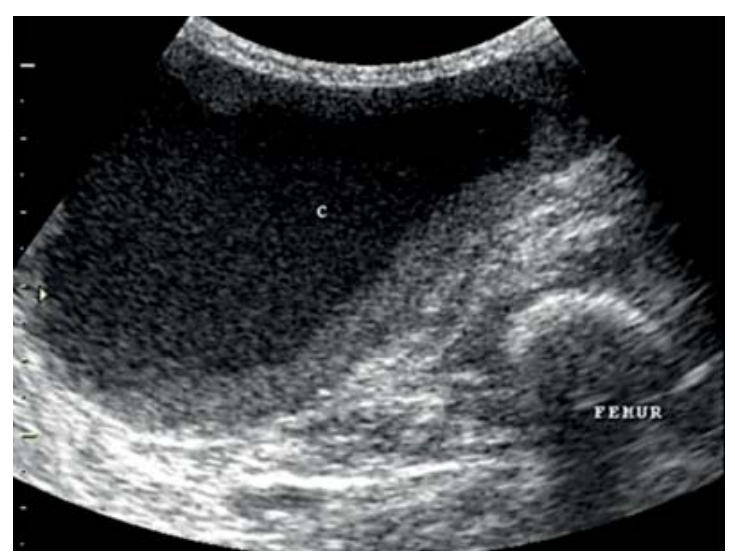

FIGURE 2: USG of abscess

on lateral and anterior faces of left thigh with liquid content and thickened walls (Figures 3 and 4).

A study of the liquid in the abscess was negative for neoplastic cells, but the Ziehl-Neelsen bacilloscopy method was positive $(+++/ 4+)$ and Gram was negative. Treatment for $M$. tuberculosis was started with rifampicin, isoniazid and pyrazinamide (RIP). The polymerase chain reaction (PCR) for the $M$. tuberculosis complex in the liquid in the abscess was negative. Chest radiography and tomography were normal.

During hospitalization, the patient evolved with headache and disorientation. The cerebrospinal fluid demonstrated 192 cells with predominance of lymphomononuclear ones. The Crypto-LA test resulted positive. Treatment for cryptococcal meningoencephalitis was started with amphotericin B deoxycholate.

Fourteen days after treatment was started following a RIP scheme with a cumulative dose of $850 \mathrm{~g}$ of amphotericin B, antiretroviral therapy (ART) was

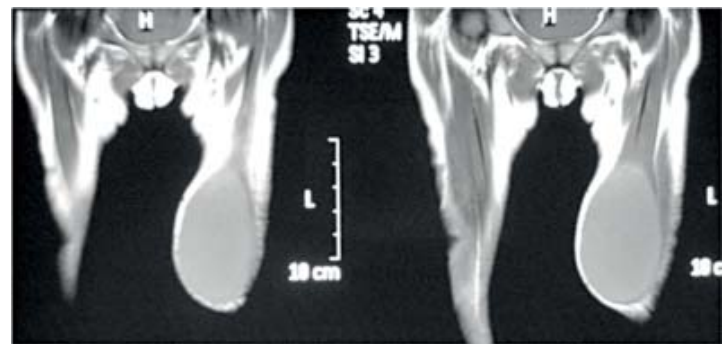

FigURE 3: MRI of lesion

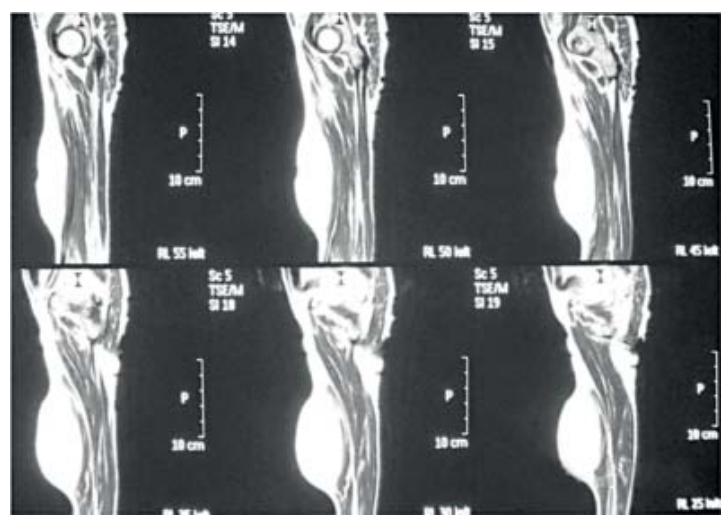

FIGURE 4: MRI of lesion

initiated with zidovudine, lamivudine and efavirenz. ART was initiated two weeks later, with the goal of diminishing the development of Immune reconstitution inflammatory syndrome (IRIS).

Mycobacteria culture in a Lowestein-Jensen medium demonstrated growth of non-tuberculous bacteria, with bright yellow colonies (chromogenic) - M. Kansasii. The isolated mycobacterium resistance profile demonstrated sensitivity to rifampicin and ethambutol and resistance to isoniazid and streptomycin. The scheme was changed to rifampicin, ethambutol, moxifloxacin and clarithromycin. The patient progressed to clinical improvement, being discharged at the $51^{\text {st }}$ day of hospitalization with a TCD4 lymphocyte count of 350 cells $/ \mu$ land undetectable viral load.

\section{DISCUSSION}

Generally speaking, the infection by M. kansasii in patients with AIDS results in pulmonary or disseminated involvement, for the lungs are the main route of entry for this bacterium. It usually occurs in patients with CD4 lower than 100 cells / $\mu 1 ., 6$ The patient described did not present clinical-radiological evidence of pulmonary involvement.

Cutaneous involvement is rare and usually occurs in patients with advanced immunodepression with disseminated disease. ${ }^{78}$ In the referred literature, 
we found a single report of cutaneous abscess by $M$. kansasii secondary to IRIS in a patient with AIDS, which demonstrates the rarity of this report. ${ }^{3}$

The direct research of the material found in the abscess demonstrated acid-fast bacilli, suggesting infection by $M$. tuberculosis, which is one of the most frequent infections in patients with AIDS in Brazil. ${ }^{1}$ For this reason, in spite of negative PCR for the $M$. tuberculosis complex, we initiated treatment using the RIP scheme.

The culture of the material found in the abscess confirmed the presence of non-tuberculous mycobacterium M. kansasii. Antibiogram demonstrated resistance of the microorganism to pyrazinamide and isoniazid, a resistance profile commonly observed in $M$. kansasii(1). Although the patient had undergone treatment with only one effective drug (rifampicin) against M. kansasii, she presented clinical improvement. At the time, ethambutol was not part of the tuberculosis treatment scheme in Brazil. ${ }^{1}$ We associated ethambutol, clarithromycin and moxifloxacin to the scheme. Clarithromycin and quinolones have demonstrated efficacy against $M$. kansasii. ${ }^{9,10}$ Treatment is recommended for a period of 18 months. ${ }^{1}$

In Brazil, in spite of high prevalence of infection by $M$. tuberculosis, the spread of infections caused by non-tuberculous mycobacteria is increasing. The importance of etiological diagnosis obtained from the culture, as well as the role of antibiogram for the correct treatment of these infections is emphasized.

\section{REFERENCES}

1. Pappalardo MCSM, Aires EM, Moreira IM, Boszczowski I, Campéas AE, Filho AMB. Micobacteriose disseminada por mycobacterium kansasii associada à aids: Relato de caso. Rev Panam Infectol. 2004;6:51-4.

2. Mitha M, Naicker P, Taljaard J. Cutaneous Mycobacterium kansasii in a patient with AIDS post initiation of antiretroviral therapy. J Infect Dev Ctries. 2011;5:553-5.

3. Connick E, Levi ME. Mycobacterium kansasii cutaneous abscess ocorring as Immune reconstitution inflamatory syndrome. Int J Tuberc Lung Dis. 2011;15:993.

4. Yano T, Okuda S, Kato K, Kato K, Kishimoto T. Mycobacterium kansasii osteomyelites in a patient with AIDS on highly active antiretroviral therapy. Intern Med. 2004;43:1084-6.

5. Manfredi R, Nanetti A, Valentini R, Ferri M, Morelli S, Calza L. Epidemiological, clinical and therapeutic features of AIDS-related Mycobacterium kansasii infection during the HIV pandemic: on 11-year-follow-up study. HIV Med. 2004;5:431-6.

6. Mathew SD, Tully CC, Borra H, Berven MD, Arroyo R. Septic subacromial bursitis caused by Mycobacterium kansasii in an immunocompromised host. Mil Med. 2012;177:617-20.

7. Han SH, Kim KM, Chin BS, Choi SH, Lee HS, Kim MS, et al. Disseminated Mycobacterium kansasii infection associated with skin lesions: A case report and comprehensive Review of the literature . J Korean Med Sci. 2010;25:304-8.

8. Cardoso F, Ramos H, Lobo M. Dermatoses in HIV-infected patients with different degrees of immunosuppression. An Bras Dermatol. 2002;77:669-80.

9. Tartaglione T. Treatment of nontuberculous mycobacterial infections, role of clarithromycin and azithromycin. Clin Ther. 1997;19:626-38.

10. Tompkins JC, Witzig RS. Mycobacterium kansasii in HIV patients: clarithromycin and antiretroviral effects. Int J Tuberc Lung Dis. 2007;11:331-7.

\author{
MAILING ADDRESS: \\ Catarina Tenório de Lima \\ Universidade Federal de Pernambuco \\ Av.Moraes Rego , n. 1235 \\ Cidade Universitária \\ 50670-901 - Recife - PE \\ Brazil \\ E-mail: catarina_tl@yahoo.com.br
}

How to cite this article: Lima CT, Magalhães V. Abscess resulting from Mycobacterium kansasii in the left thigh of AIDS patient. An Bras Dermatol. 2014;89(3):478-80. 